\title{
Obstacle avoidance method of three-dimensional obstacle spherical cap
}

\author{
YANG Xiuxia", ZHANG Yi, and ZHOU Weiwei \\ Naval Aviation University, Yantai 264001, China
}

\begin{abstract}
Focusing on obstacle avoidance in three-dimensional space for unmanned aerial vehicle (UAV), the direct obstacle avoidance method in dynamic space based on three-dimensional velocity obstacle spherical cap is proposed, which quantifies the influence of threatening obstacles through velocity obstacle spherical cap parameters. In addition, the obstacle avoidance schemes of any point on the critical curve during the multi-obstacles avoidance are given. Through prediction, the insertion point for the obstacle avoidance can be obtained and the flight path can be replanned. Taking the Pythagorean Hodograph $(\mathrm{PH})$ curve trajectory re-planning as an example, the three-dimensional direct obstacle avoidance method in dynamic space is tested. Simulation results show that the proposed method can realize the online obstacle avoidance trajectory re-planning, which increases the flexibility of obstacle avoidance greatly.
\end{abstract}

Keywords: three-dimensional direct space obstacle avoidance, velocity obstacle cone, velocity obstacle spherical cap.

DOI: $10.21629 /$ JSEE.2018.05.16

\section{Introduction}

The online planning in non-structural environment should contain the avoidance of unknown danger, so the threat of collision should be predicted and effective collision avoidance re-planning should be carried out. The detection information mainly includes the distance between unmanned aerial vehicle (UAV) and obstacle, direction, sight angle, sight angular velocity, the relative speed and acceleration. There are two main types of obstacle avoidance. One is to add the position information of the UAV and the obstacles as constrains into the trajectory planning, such as A*search algorithm, fast random search tree [1], genetic algorithm [2], particle swarm optimization (PSO) algorithm [3-5], fluid flow avoiding obstacles method [6], and artificial potential field method [7] which removes the solution that does not meet the obstacle avoidance constraint. The other

Manuscript received May 27, 2017

*Corresponding author.

This work was supported by the Aeronautical Science Foundation of China (20135584010). is to design the obstacle avoidance guidance law according to direction and position. The first method is mainly used for static obstacle avoidance. For the dynamic planning, the second method is generally used to meet the reaction time and rapid mobility requirements which can fully uses the motion information and relative motion trend of UAV and obstacle [8]. Now, more and more scholars pay attention to the latter method [9-11].

Shin et al. [12-14] conducted a research on real-time collision detection and obstacle avoidance method of dynamic obstacle during the flight based on the differential geometry, and constructed collision detection with the velocity obstacle cone method and collision resolution with the guidance law. The guidance law in this paper is noncontinuous and its planning path is prone to flutter.

Anusha et al. [15] gave two nonlinear obstacle avoidance guidance methods: nonlinear geometric guidance method and differential geometric guidance method, thus the UAV can rapidly align the velocity vector with the target point and present the corresponding guidance method.

Chen et al. [16] proposed a new collision avoidance guidance method combining the tangent vector guidance method with the Lyapunov vector guidance method, where the tangent vector guidance method is adopted if tangent vector exists, otherwise, the Lyapunov vector guidance method is adopted, which is used in two-dimensional collision avoidance.

Now, some papers concentrate on dynamic obstacle avoidance in three-dimensional space.

For the problem of three-dimensional space obstacles avoidance, in [17], according to the relative movement of the UAV and the obstacle, the iterative method was used to determine the obstacles avoiding direction, which has bad real time performance.

In $[18,19]$, the idea of dimensionality reduction was used in the three-dimensional space obstacles avoidance. In [18], the proportional guidance was applied in the vertical plane or the horizontal plane according to the component of the relative velocity of the UAV and the obsta- 
cle. Belkhouche [19] realized the three-dimensional obstacle avoidance with the obstacle cone method based on the plane dimension reduction. The extended proportional navigation law is used to complete the avoidance guidance. The constraints such as velocity and angular velocity have been taken into consideration in the parameter design, which can create non-straight line trajectory. The dimension reduction obstacle avoidance method will cause the problem as large flight angle shift and large deviation from the original track and so on.

Based on the proportional guidance minimum maneuver strategy, in [20], the obstacle avoidance method was provided for input change and avoiding multiple obstacles, which is suitable for static obstacle and cannot settle the dynamic obstacle avoidance.

In $[21,22]$, the definition of three-dimensional velocity obstacle cone was proposed. A plane in the space is selected as the deconflicting plane before the rotation angle is figured out through the UAV and obstacle velocity projection, which is not easy to handle the simultaneous multiple obstacles.

This paper contributes to the literature by proposing a novel three-dimensional velocity obstacle method, which has several advantages over other methods. Firstly, the concept of velocity obstacle spherical cap of UAV is introduced to the velocity obstacle method, which quantifies the influence of threatening obstacles. The method is designed to handle simultaneous multiple dynamics obstacles avoidance, and the UAV velocity vector direction for obstacles avoidance can be gotten. Secondly, it applies to the direct UAV velocity vector arbitrary change but not the dimension reduction method $[18,19]$ or the selective avoidance plane method [21,22], which satisfies different performance indexes and task requirements, and improves the obstacle avoidance flexibility. Additional contribution of this paper is, through prediction, the insertion point position of UAV re-planed flight path for the obstacles avoidance can be obtained. With this insertion point position and velocity vector direction for obstacles avoidance, the flight path of UAV can be re-planned.

This paper is structured as follows. After this introduction, the second section discusses the establishment of three-dimensional velocity obstacle cone and shows the method of collision detection. Section 3 explains the concept of the space velocity obstacle spherical cap and its parameters solution. Section 4 presents the solution method for the single and multiple dynamic obstacles avoidance direction and insertion point. Section 5 presents the implementation of the three-dimensional velocity obstacle (VO) method in simulations. Here, several encounter cases, as well as choice of avoidance are simulated and discussed. Section 6 ends the paper with several concluding remarks.

\section{Establishment and collision judgment of three-dimensional velocity obstacle cone}

As to the UAV in three-dimensional space, in the inertial coordinate system $\{X, Y, Z\}$, suppose the velocity of UAV is $\boldsymbol{v}_{u}(t)$ at $t$, its motion state is defined as $U(t)=\left(P_{u}(t), \boldsymbol{v}_{u}(t)\right)$, where $P_{u}(t)=\left(x_{u}, y_{u}, z_{u}\right)$ is position, $\boldsymbol{v}_{u}(t)$ is the velocity vector of UAV (as shown in Fig. 1). In a planning period $T$, suppose the velocity of UAV remains unchanged. $O$ is the obstacle, the position is $P_{o}(t)=\left(x_{o}, y_{o}, z_{o}\right)$, the velocity is $\boldsymbol{v}_{o}(t)$. According to the relative size of the relationship between UAV and obstacle, the UAV can be simplified to a bit and the obstacle can be seen as a sphere, the radius of obstacle is $R$, and the swelled obstacle is called position obstacle (PO) of UAV.

A space velocity collision cone $(\mathrm{CC})$ can be derived by dropping tangents from the UAV to the obstacle ball, which is shown in Fig. 1. The apex of $\mathrm{CC}$ is the UAV position $P_{U}$, where every tangential line from $P_{U}$ to the edge of $\mathrm{PO}$ is a generating line of the cone.

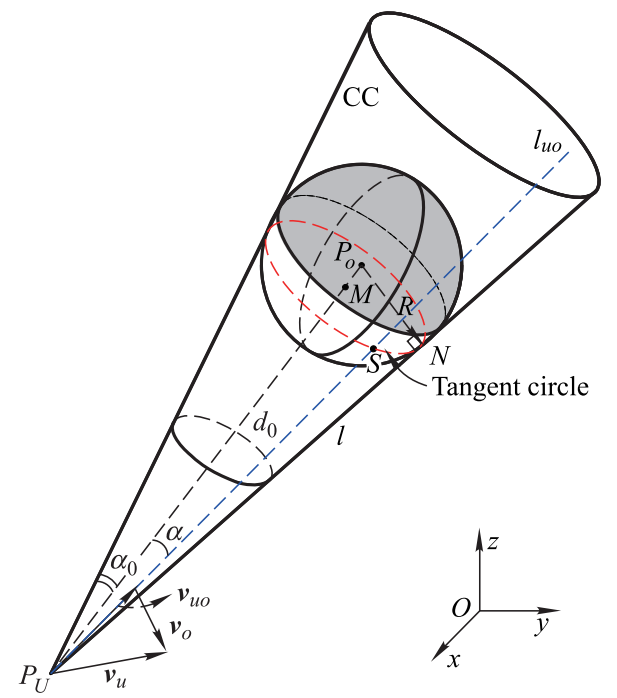

Fig. 1 Schematic diagram of three-dimensional space $\mathrm{CC}$

The relative velocity vector between UAV and obstacle can be expressed as

$$
\boldsymbol{v}_{u o}=\boldsymbol{v}_{u}-\boldsymbol{v}_{o} .
$$

Through the relative velocity vector, $O$ can be regarded as static obstacle and the velocity of UAV can be considered as $\boldsymbol{v}_{u o} . l_{u o}$ is the line on the direction of the relative velocity vector $\boldsymbol{v}_{u o}$, if $\boldsymbol{v}_{u o}$ remains unchanged, the collision condition between $\mathrm{UAV}$ and obstacle $O$ is

$$
l_{u o} \cap \mathrm{PO} \neq \varnothing .
$$

The set that meets (2) is called three-dimensional relative $\mathrm{CC}$ ( $\mathrm{RCC})$.

$$
\mathrm{RCC}=\left\{\boldsymbol{v}_{u o} \mid l_{u o} \cap \mathrm{PO} \neq \varnothing\right\}
$$


As is shown in Fig. 1, if $\boldsymbol{v}_{u o} \in \mathrm{RCC}$, UAV will collide with the obstacle.

The grey area shown in Fig. 2 can be formed by translating RCC $\left\|\boldsymbol{v}_{o}\right\|$ along the direction of $\boldsymbol{v}_{o}$, which is called absolute $\mathrm{CC}$ (ACC):

$$
\mathrm{ACC}=\mathrm{RCC} \oplus \boldsymbol{v}_{o}
$$

where, $\oplus$ is the Minkowski vector sum.

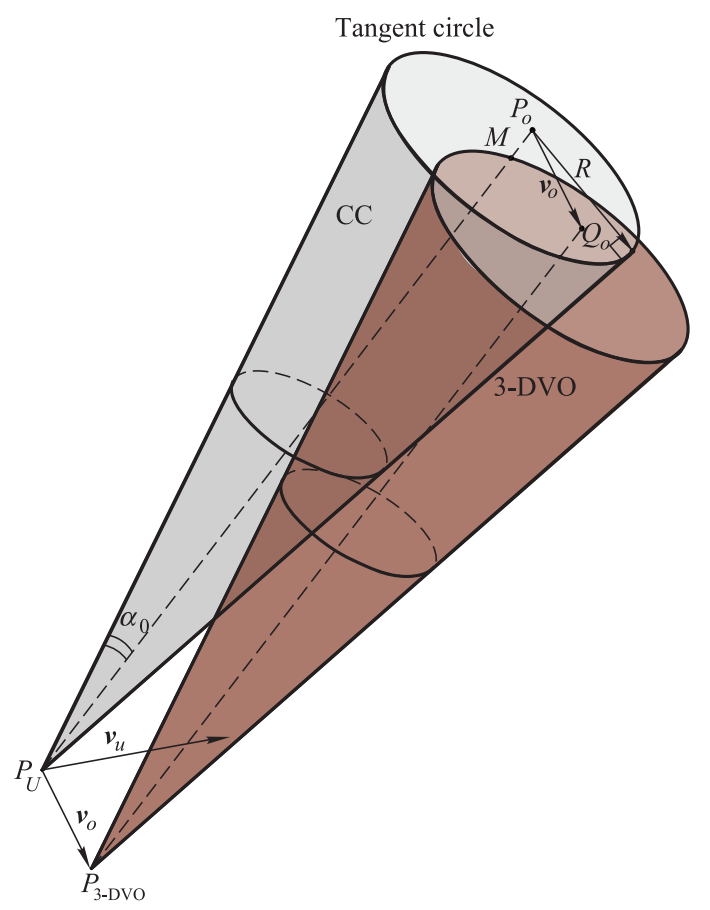

Fig. 2 Schematic diagram of three-dimensional space VO cone

It can be seen that if $\boldsymbol{v}_{u} \in \mathrm{ACC}, \boldsymbol{v}_{u o} \in \mathrm{RCC}$ and UAV will collide with the obstacle, the corresponding velocity $\boldsymbol{v}_{u}$ is called three-dimensional velocity obstacle (3-DVO). The steps of collision detection are as follows:

Step 1 Calculate the semi-apex angle $\alpha_{0}$ of obstacle cone according to the detection distance $d_{0}$ of UAV and the swelled radius $R$ of obstacle

$$
\alpha_{o}=\arcsin \frac{R}{d_{0}} .
$$

Step 2 Determine the position $P_{u}\left(x_{u}, y_{u}, z_{u}\right)$ of UAV and $P_{o}\left(x_{o}, y_{o}, z_{o}\right)$ of obstacle $O$ according to the corresponding sensor information, then the angle between the relative velocity vector $\boldsymbol{v}_{u o}$ and position vector $\boldsymbol{P}_{u} \boldsymbol{P}_{o}$ can be drawn as

$$
\begin{gathered}
\cos \alpha=\cos \left\langle\boldsymbol{v}_{u o}, \boldsymbol{P}_{u} \boldsymbol{P}_{o}\right\rangle= \\
\frac{v_{u o x}\left(x_{o}-x_{u}\right)+v_{u o y}\left(y_{o}-y_{u}\right)+v_{u o z}\left(z_{o}-z_{u}\right)}{\left\|\boldsymbol{v}_{u o}\right\|\left\|\boldsymbol{P}_{u} \boldsymbol{P}_{o}\right\|}
\end{gathered}
$$

where

$$
\left\|\boldsymbol{P}_{u} \boldsymbol{P}_{o}\right\|=\sqrt{\left(x_{o}-x_{u}\right)^{2}+\left(y_{o}-y_{u}\right)^{2}+\left(z_{o}-z_{u}\right)^{2}} .
$$

Step 3 According to $\alpha$ and $\alpha_{0}$, the judgment method of collision avoidance can be obtained as

(i) If $\alpha<\alpha_{0}$, it indicates that the relative velocity vector is in the obstacle cone, so the UAV needs to avoid the detected obstacle;

(ii) If $\alpha>\alpha_{0}$, it shows that the relative velocity vector is not in the obstacle cone, so the UAV does not need to avoid the detected obstacle.

$\alpha \in[0, \pi / 2)$, when the $\alpha$ reaches $\pi / 2$, the UAV position is exactly on the surface of the zone $\mathrm{PO}$, and $d_{0}=R$. A degenerate case happens when the two vehicles collide, or when $d_{0}<R$, in which case the VO cone cannot be defined.

The condition of UAV's collision to multiple obstacles is that the conditions of UAV's collision avoidance to single obstacle are all satisfied, that is

$$
\alpha_{i}<\alpha_{o_{i}}, \quad \forall i \in\{1,2, \ldots, n\} .
$$

That is the relative velocity vector $\boldsymbol{v}_{\text {uoi }}$ between obstacle $O_{i}$ and velocity vector $\boldsymbol{v}_{u}$ are all located in the obstacle cone of $O_{i}$.

The condition of UAV's collision avoidance to every obstacle is

$$
\alpha_{i} \nless \alpha_{o_{i}}, \quad \forall i \in\{1,2, \ldots, n\} .
$$

\section{Establishment and parametric solution of space VO spherical cap (SC)}

\subsection{Establishment of the space VOSC}

When UAV avoids the threat obstacle, keep the size of the velocity vector $\boldsymbol{v}_{u}$ constant, change the direction of the velocity vector $\boldsymbol{v}_{u}$ of UAV. For the realization of the UAV's collision avoidance of obstacles, UAV's velocity vector needs to be rotated out 3-DVO. Fig. 3 shows the schematic diagram of velocity sphere (VS) and 3-DVO, the part located within the 3-DVO is called SC, namely for the red part on VS.

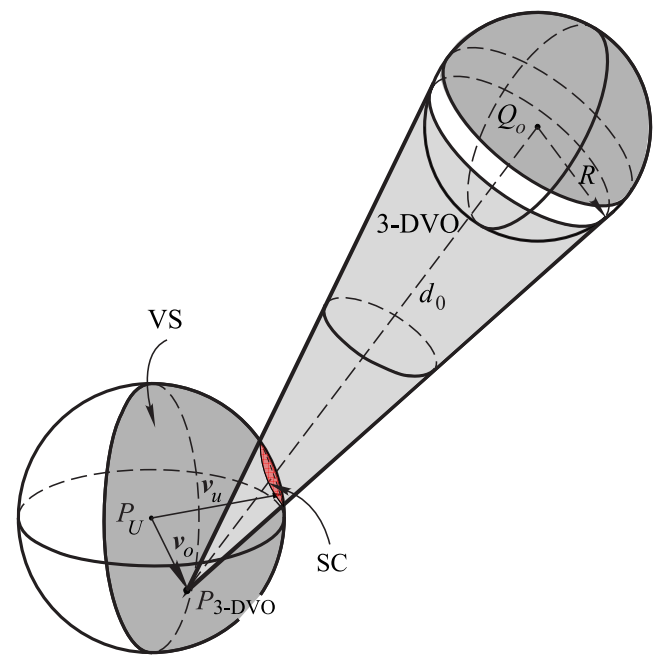

Fig. 3 Schematic diagram of UAV's VOSC 
Then, denote the SC as $G_{o}=\left(r, \psi_{r}, \theta_{r}, \gamma\right)$, among them, $r$ is the length of the vector $\boldsymbol{P}_{u} \boldsymbol{M}$, which is formed by the SC center and the ball center; $\gamma$ is half-apex angle, which is formed by UAV's coordinates of point $P_{u}$ and $M$, $\gamma \in[0, \pi / 2]$, schematic diagram is shown in Fig. 4. Therefore, threat obstacle's impact on the UAV's flight is translated into SC on VS, so the research on the UAV collision avoidance of threat obstacle is translated into the research on the size and direction of the SC on VS. This idea of translation greatly simplifies the model of threat obstacle avoidance in three-dimensional space, quantifies the impact of threat obstacle. The effect of non-threat obstacle can also be considered.

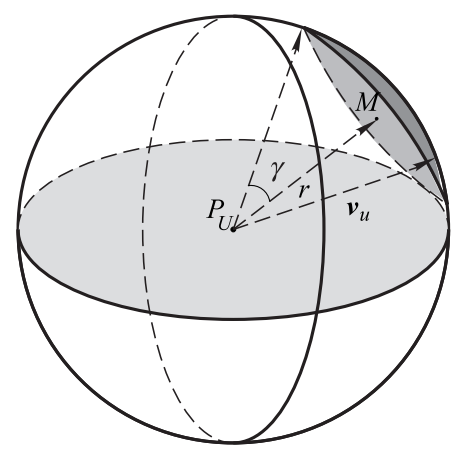

Fig. 4 Size and direction schematic diagram of SC formed by obstacles

\subsection{Parametric solution of the space VOSC}

The key of parametric solution of the space VOSC is to solve the collision avoidance critical point, which goes through the vector $\boldsymbol{P}_{3 \text {-DVO }} \boldsymbol{Q}_{o}$ of space plane, and collision avoidance critical state point is in the intersecting generatrix of the space plane and space VO cone. To simplify the parametric solution of the space VOSC, plane that goes through the vector $\boldsymbol{P}_{3 \text {-DVO }} \boldsymbol{Q}_{o}$ and the relative velocity vector $\boldsymbol{v}_{u o}$ (hereinafter referred to as collision avoidance plane) is selected to solve collision avoidance critical point on the intersecting generatrix of the collision plane and space VO cone.

\subsubsection{Critical state solution of $\boldsymbol{v}_{u}$ 's minimum turning angle when UAVs avoid the obstacle}

Construct a plane $P_{3-\mathrm{DVO}} Q_{o} T$ according to space vector $\boldsymbol{v}_{u o}$ and $\boldsymbol{P}_{3-\mathrm{DVO}} \boldsymbol{Q}_{o}$, then generatrix $l_{3}$ is the intersection of plane $P_{3-\mathrm{DVO}} Q_{o} T$ and cone surface $P_{3 \text {-DVO }} l_{1} l_{2}$, generatrix $l_{3}$ is tangential with obstacle at point $Q$. (As shown in Fig. 5)

And, in order to insure UAV to avoid the detected obstacle with the optimum strategy, the direction of the desired relative velocity $\hat{\boldsymbol{v}}_{u o}$ is determined by rotating relative velocity $\boldsymbol{v}_{u o}$ to the direction of generatrix $l_{3}$ in the plane $P_{3 \text {-DVo }} Q_{o} T$, then the rotation angle $\delta$ can be determined as

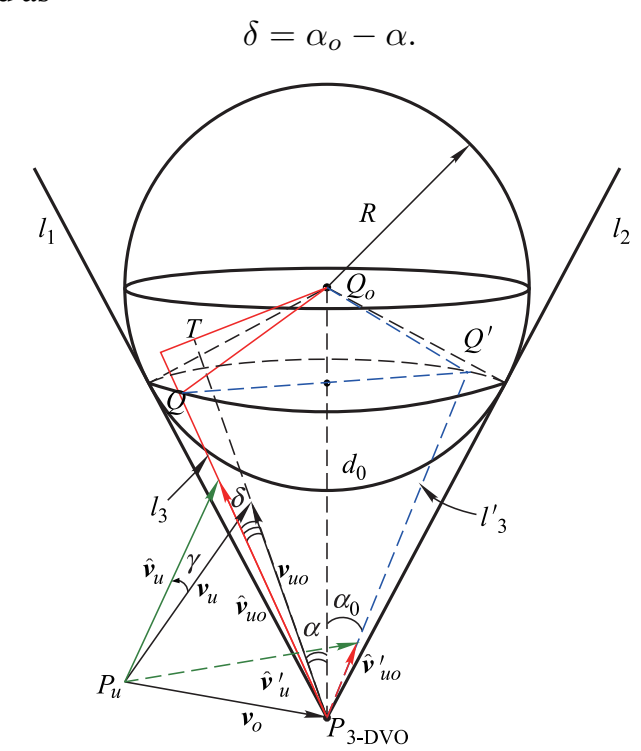

Fig. 5 Schematic diagram of minimum and maximum rotation angle of UAV's velocity vector $\boldsymbol{v}_{u}$

To determine the drift angle $\Delta \psi_{u o}$ and pitch angle $\Delta \theta_{u o}$ during the process of rotating relative velocity vector $\boldsymbol{v}_{u o}$ to the direction of generatrix $l_{3}$, a unit vector $\boldsymbol{q}$ on the generatrix $l_{3}$ needs to be determined. Select a point $P\left(x_{P}, y_{P}, z_{P}\right)$ and make $\left\|\boldsymbol{P}_{u} \boldsymbol{P}\right\|=1$. Among them, the vertex $P_{3 \text {-DVo }}\left(x_{3 \text {-DVO }}, y_{3-\mathrm{DVO}}, z_{3-\mathrm{DVO}}\right)$ of 3 -DVO can be determined as

$$
P_{3-\mathrm{DVO}}=P_{u}+\boldsymbol{v}_{o} .
$$

According to the geometric relationship in Fig. 5, the unknown point can be solved, and the corresponding geometrical relation can be obtained as

$$
\begin{gathered}
\left\|\boldsymbol{P}_{3-\mathrm{DVO}} \boldsymbol{P}\right\|=1 \\
\cos \delta=\frac{\boldsymbol{v}_{u o} \cdot \boldsymbol{P}_{3-\mathrm{DVO}} \boldsymbol{P}}{\left\|\boldsymbol{v}_{u o}\right\|\left\|\boldsymbol{P}_{3-\mathrm{DVO}} \boldsymbol{P}\right\|} \\
\cos \alpha_{0}=\frac{\boldsymbol{P}_{3-\mathrm{DVO}} \boldsymbol{P} \cdot \boldsymbol{P}_{3-\mathrm{DVO}} \boldsymbol{Q}_{o}}{\left\|\boldsymbol{P}_{3-\mathrm{DVO}} \boldsymbol{P}\right\|\left\|\boldsymbol{P}_{3-\mathrm{DVO}} \boldsymbol{Q}_{o}\right\|} .
\end{gathered}
$$

Therefore, the coordinates of point $P$ can be obtained. Then the unit vector $\boldsymbol{q}$ on generatrix $l_{3}$ can be expressed as

$$
\boldsymbol{q}=\left(x_{P}-x_{3-\mathrm{DVO}}, y_{P}-y_{3-\mathrm{DVO}}, z_{P}-z_{3 \text {-DVO }}\right) .
$$

The desired relative velocity $\hat{\boldsymbol{v}}_{u o}$ can be expressed as

$$
\hat{\boldsymbol{v}}_{u o}=\lambda \boldsymbol{q} .
$$

Among them, $\lambda$ is the size of desired relative velocity vector $\hat{\boldsymbol{v}}_{u o}$.

As the size and direction of obstacle's velocity vector $\boldsymbol{v}_{o}$ are known, the included angle between the obstacle's 
velocity vector $\boldsymbol{v}_{o}$ and the desired relative velocity vector $\hat{\boldsymbol{v}}_{u o}$ can be written as

$$
\cos \left\langle\boldsymbol{v}_{o}, \hat{\boldsymbol{v}}_{u o}\right\rangle=\frac{\boldsymbol{v}_{o} \cdot(\lambda \boldsymbol{q})}{\left\|\boldsymbol{v}_{o}\right\|\left\|\hat{\boldsymbol{v}}_{u o}\right\|}=\frac{\boldsymbol{v}_{o} \cdot \boldsymbol{q}}{\left\|\boldsymbol{v}_{o}\right\|}
$$

From Fig. 5, the size of desired relative velocity vector $\hat{\boldsymbol{v}}_{u o}$ meets the following equation:

$$
\cos \left(\pi-\left\langle\boldsymbol{v}_{o}, \hat{\boldsymbol{v}}_{u o}\right\rangle\right)=\frac{\left\|\boldsymbol{v}_{o}\right\|^{2}+\left\|\hat{\boldsymbol{v}}_{u o}\right\|^{2}-\left\|\boldsymbol{v}_{u}\right\|^{2}}{2\left\|\boldsymbol{v}_{o}\right\|\left\|\hat{\boldsymbol{v}}_{u o}\right\|} .
$$

Equation (13) has two solutions. According to Fig. 5, the size of $\hat{\boldsymbol{v}}_{u o}$ can be determined as

$$
\begin{gathered}
\hat{\boldsymbol{v}}_{u o}=\left\|\boldsymbol{v}_{o}\right\| \cos \left(\pi-\left\langle\boldsymbol{v}_{o}, \hat{\boldsymbol{v}}_{u o}\right\rangle\right)+ \\
\sqrt{\left\|\boldsymbol{v}_{o}\right\|^{2} \cos ^{2}\left(\pi-\left\langle\boldsymbol{v}_{o}, \hat{\boldsymbol{v}}_{u o}\right\rangle\right)-\left(\left\|\boldsymbol{v}_{o}\right\|^{2}-\left\|\boldsymbol{v}_{u}\right\|^{2}\right)} .
\end{gathered}
$$

Based on the velocity vector triangle,

$$
\hat{\boldsymbol{v}}_{u}=\boldsymbol{v}_{o}+\hat{\boldsymbol{v}}_{u o}
$$

can be obtained. Besides,

$$
\boldsymbol{v}_{o}=\left[\left\|\boldsymbol{v}_{o}\right\| \cos \theta_{o} \cos \psi_{o},\left\|\boldsymbol{v}_{o}\right\| \cos \theta_{o} \sin \psi_{o},\left\|\boldsymbol{v}_{o}\right\| \sin \theta_{o}\right]
$$

$\hat{\boldsymbol{v}}_{u o}=\left[\lambda\left(x_{P}-x_{3-\mathrm{DVO}}\right), \lambda\left(y_{P}-y_{3-\mathrm{DVO}}\right), \lambda\left(z_{P}-z_{3-\mathrm{DVO}}\right)\right]$.

Thus, the desired relative velocity vector of UAV can be expressed as

$$
\begin{gathered}
\hat{\boldsymbol{v}}_{u}=\left[\begin{array}{c}
\hat{v}_{u x} \\
\hat{v}_{u y} \\
\hat{v}_{u z}
\end{array}\right]= \\
{\left[\begin{array}{c}
\left\|\boldsymbol{v}_{o}\right\| \cos \theta_{o} \cos \psi_{o}+\left\|\hat{\boldsymbol{v}}_{u o}\right\|\left(x_{P}-x_{3-\mathrm{DVO})}\right) \\
\left\|\boldsymbol{v}_{o}\right\| \cos \theta_{o} \sin \psi_{o}+\left\|\hat{\boldsymbol{v}}_{u o}\right\|\left(y_{P}-y_{3-\mathrm{DVO}}\right) \\
\left\|\boldsymbol{v}_{o}\right\| \sin \theta_{o}+\left\|\hat{\boldsymbol{v}}_{u o}\right\|\left(z_{P}-z_{3-\mathrm{DVO}}\right)
\end{array}\right] .}
\end{gathered}
$$

Therefore, the direction angle $\left(\hat{\psi}_{u}, \hat{\theta}_{u}\right)$ of UAV's desired velocity vector can be expressed as

$$
\begin{gathered}
\cos \hat{\psi}_{u}=\frac{\hat{v}_{u x}}{\sqrt{\hat{v}_{u x}^{2}+\hat{v}_{u y}^{2}}} \\
\sin \hat{\theta}_{u}=\frac{\hat{v}_{u z}}{\sqrt{\hat{v}_{u x}^{2}+\hat{v}_{u y}^{2}+\hat{v}_{u z}^{2}}} .
\end{gathered}
$$

According to the direction angle $\left(\psi_{u}, \theta_{u}\right)$ of instant velocity vector $\boldsymbol{v}_{u}$ and the direction angle $\left(\hat{\psi}_{u}, \hat{\theta}_{u}\right)$ of desired velocity vector $\hat{\boldsymbol{v}}_{u}$, the minimum turning angle required for UAV to complete the obstacle avoidance mission can be expressed as

$$
\Delta \psi=\hat{\psi}_{u}-\psi_{u}
$$

$$
\Delta \theta=\hat{\theta}_{u}-\theta_{u} .
$$

Among them, $\Delta \psi>0$ and $\Delta \theta>0$ mean counter clockwise rotation, $\Delta \psi<0$ and $\Delta \theta<0$ mean positive rotation.

Based on the minimum turning angle $(\Delta \psi, \Delta \theta)$ of UAV's velocity vector, the relation between UAV's instant velocity vector $\boldsymbol{v}_{u}$ and the desired direction angle $\hat{\boldsymbol{v}}_{u}$ can be determined as

$$
\hat{\boldsymbol{v}}_{u}=\left[\begin{array}{cc}
\cos \Delta \theta & \sin \Delta \theta \\
-\sin \Delta \theta & \cos \Delta \theta
\end{array}\right]\left[\begin{array}{cc}
\cos \Delta \psi & \sin \Delta \psi \\
-\sin \Delta \psi & \cos \Delta \psi
\end{array}\right] \boldsymbol{v}_{u}
$$

3.2.2 Solution of maximum deflection critical point of collision avoidance plane

On the basis of Fig. 5, collision avoidance plane $P_{3 \text {-DVO }} Q Q_{o} Q^{\prime}$ intersects obstacle cone at $l_{3}^{\prime}, l_{3}^{\prime}$ is the generatrix of obstacle cone. Because the generatrix $l_{3}$ is minimum deflection position of UAV's velocity vector in the process of obstacle avoidance, $l_{3}^{\prime}$ and $l_{3}$ are coplanar, so generatrix $l_{3}^{\prime}$ corresponds to the UAV maximum position of velocity vector deflection angle in the process of obstacle avoidance. According to the method that calculates UAV's expected size and direction of the velocity vector on the generatrix $l_{3}$, the size and direction of relative velocity vector $\hat{\boldsymbol{v}}_{u o}^{\prime}$ and desired velocity vector $\hat{\boldsymbol{v}}_{u}^{\prime}$ corresponding to the generatrix $l_{3}^{\prime}$ can be calculated.

\subsubsection{Parameters solution of space VOSC}

Based on the solution of critical state point on the collision avoidance plane in Sections 3.2.1 and 3.2.2, the four unknown parameters that describe space VOSC can be solved. The velocity vector diagram of collision avoidance that UAV avoids obstacle $O$ in Fig. 5 can be abstracted as Fig. 6. Among them, $B$ and $C$ are critical points on the critical edges $l_{3}$ and $l_{3}^{\prime}$.

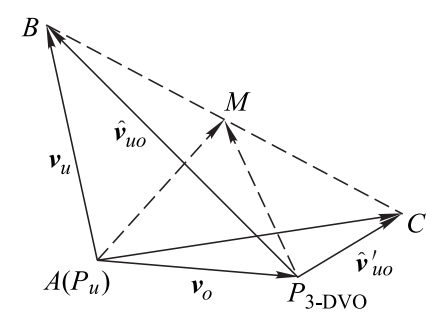

Fig. 6 Vector relation diagram between UAV and obstacle $O$ in three-dimensional space

In Fig. 6, $M$ is the midpoint of segment $B C$. Based on the solving magnitude and direction of desired velocity vectors $\hat{\boldsymbol{v}}_{u o}, \hat{\boldsymbol{v}}_{u o}^{\prime}$ and the addition principle of vector triangle, it can be obtained

$$
\boldsymbol{P}_{3-\mathrm{DVO}} \boldsymbol{M}=\frac{1}{2}\left(\boldsymbol{P}_{3-\mathrm{DVO}} \boldsymbol{B}+\boldsymbol{P}_{3-\mathrm{DVO}} \boldsymbol{C}\right)=\frac{1}{2}\left(\hat{\boldsymbol{v}}_{u o}+\hat{\boldsymbol{v}}_{u o}^{\prime}\right) \text {. }
$$


Then, the vector $\boldsymbol{A} \boldsymbol{M}$ can be written as

$$
\begin{gathered}
\boldsymbol{A} \boldsymbol{M}=\boldsymbol{A} \boldsymbol{P}_{3-\mathrm{DVO}}+\boldsymbol{P}_{3-\mathrm{DVO}} \boldsymbol{M}=\boldsymbol{v}_{o}+\frac{1}{2}\left(\hat{\boldsymbol{v}}_{u o}+\hat{\boldsymbol{v}}_{u o}^{\prime}\right)= \\
\left(x_{A M}, y_{A M}, z_{A M}\right) .
\end{gathered}
$$

Thus, the length $r$ and direction angle $\left(\psi_{r}, \theta_{r}\right)$ of vector $\boldsymbol{A} \boldsymbol{M}$ can be written as

$$
\begin{gathered}
\cos \psi_{r}=\frac{x_{A M}}{\sqrt{x_{A M}^{2}+y_{A M}^{2}}} \\
\sin \theta_{r}=\frac{z_{A M}}{\sqrt{x_{A M}^{2}+y_{A M}^{2}+z_{A M}^{2}}} .
\end{gathered}
$$

Besides, the parameter $\gamma$ that describes the size of SC $G_{O}$ can be determined as

$$
\sin \gamma=\frac{\|\boldsymbol{B} \boldsymbol{C}\|}{2\left\|\boldsymbol{v}_{u}\right\|} .
$$

Thus, four parameters $\gamma, \psi_{r}, \theta_{r}, \gamma$ that describe the size and direction of SC $G_{O}$ can be solved, and the influence of threat obstacle to UAV can be quantified by solving SC parameters.

\section{Solution of obstacle avoidance direction and obstacle avoidance point based on the space VOSC}

\subsection{Solution of collision avoidance direction of single threat obstacle}

The simplest threat obstacle avoidance situation is single obstacle avoidance. Rotating the velocity vector of the UAV out of the three-dimensional space VO can realize UAV's collision avoidance of single threat obstacle, rotating the relative velocity vector between the UAV and the obstacle to the generatrix of three-dimensional space CC. In the process of solving space obstacle SC's parameters, the rotating direction of the minimum space velocity vector should be determined, considering the direction of the critical velocity vector $\hat{\boldsymbol{v}}_{u}$ that corresponds to the velocity vector $\boldsymbol{v}_{u}$ at minimum space rotation angle as the velocity vector direction of single threat obstacle collision avoidance.

\subsection{Solution of collision avoidance direction of multiple threat obstacles}

Below is an example of two threat obstacles to solve the velocity vector direction of multiple threat obstacles collision avoidance. Similar solving method can be used to solve the velocity vector direction of three or more threat obstacles collision avoidance. Fig. 7 shows the threat obstacle SC that corresponds to two threat obstacles, according to the parametric solution of space VOSC, the calculated SC parameters of obstacles $O_{1}$ and $O_{2}$ respectively are $G_{o_{1}}\left(r_{1}, \psi_{r_{1}}, \theta_{r_{1}}, \gamma_{1}\right)$ and $G_{o_{2}}\left(r_{2}, \psi_{r_{2}}, \theta_{r_{2}}, \gamma_{2}\right)$.
Among them, $r_{1}$ and $r_{2}$ respectively are the size of SC vectors $\boldsymbol{P}_{u} \boldsymbol{M}_{1}$ and $\boldsymbol{P}_{u} \boldsymbol{M}_{2},\left(\psi_{r_{1}}, \theta_{r_{1}}\right)$ and $\left(\psi_{r_{2}}, \theta_{r_{2}}\right)$ are direction angles of SC vectors $\boldsymbol{P}_{u} \boldsymbol{M}_{1}$ and $\boldsymbol{P}_{u} \boldsymbol{M}_{2}, \gamma_{1}$ and $\gamma_{2}$ respectively are the sizes of the SCs $G_{o_{1}}$ and $G_{O_{2}}$.

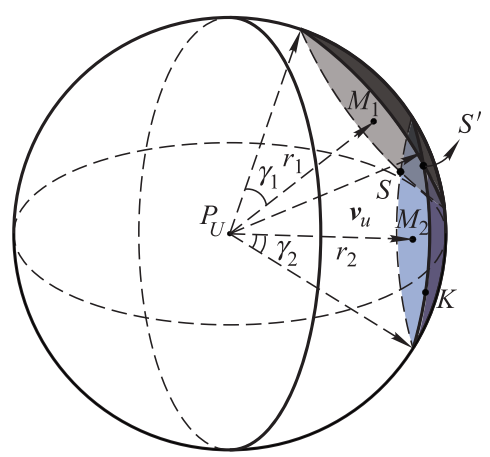

Fig. 7 Schematic diagram of multiple threat space VOSC

In Fig. 7, the space VOSC is formed by two threat obstacles intersects, and the velocity vector $\boldsymbol{v}_{u}$ of UAV locates in the intersecting public area of two space VOSC. Points $S$ and $S^{\prime}$ respectively are intersection points of two greatest circles of the $\mathrm{SC}$.

\subsubsection{Solution of obstacle avoidance direction of critical points $S$ and $S^{\prime}$}

When the UAV avoids two threat obstacles simultaneously, rotate the velocity vector $\boldsymbol{v}_{u}$ to vectors $\boldsymbol{P}_{u} \boldsymbol{S}$ and $\boldsymbol{P}_{u} \boldsymbol{S}^{\prime}$, and UAV can realize the collision avoidance of two threat obstacles; when the number of obstacles are three or more, it needs to check whether the intersection point of space VOSC is on all space velocity SC or not, to determine whether rotating the velocity vector $\boldsymbol{v}_{u}$ to the intersection point can realize collision avoidance of multiple threat obstacles.

The collision avoidance velocity vector that corresponds to the intersection point of space VOSC can be determined according to the vector relation diagram in Fig. 7,

$$
\begin{aligned}
& \boldsymbol{P}_{u} \boldsymbol{M}_{1} \perp \boldsymbol{M}_{1} \boldsymbol{S} \\
& \boldsymbol{P}_{u} \boldsymbol{M}_{2} \perp \boldsymbol{M}_{2} \boldsymbol{S} .
\end{aligned}
$$

Because the point $S$ is on UAV's velocity sphere $S_{P_{u}}$, then

$$
\left\|\boldsymbol{P}_{u} \boldsymbol{S}\right\|=v_{u}
$$

By (29) - (31), the coordinates of critical state point $S$ of UAV's obstacle avoidance can be solved, and the coordinates of $S^{\prime}$ can be solved similarly. Then, the expected velocity vector $\hat{\boldsymbol{v}}_{u}$ corresponding to the UAV obstacle avoidance critical state points $S$ and $S^{\prime}$ can be determined.

\subsubsection{Solution of obstacle avoidance direction of arbitrary point on the critical boundary}

Obstacle avoidance critical state points $S$ and $S^{\prime}$ deter- 
mined in section 4.2.1 can be used as a choice for velocity vector direction of multiple threat obstacles collision avoidance. In the process of UAV's collision avoidance of multiple threat obstacles, boundary of union set of VOSC can be used as a choice for velocity vector direction of UAV's obstacle avoidance, and any point $K$ on the obstacle avoidance boundary can be chosen as the critical state point for obstacle avoidance.

Thus according to the requirements of the task, the UAV can choose any point of the critical curve for obstacle avoidance. Assume any point of the critical curve of SC $G_{o_{2}}$ is $K\left(x_{k}, y_{k}, z_{k}\right)$ as the geometric position relationship shown in Fig. 7.

$$
\begin{gathered}
\left|\boldsymbol{P}_{u} \boldsymbol{K}\right|=v_{u} \\
\boldsymbol{P}_{u} \boldsymbol{M}_{2} \perp \boldsymbol{M}_{2} \boldsymbol{K}
\end{gathered}
$$

Relational expression of three coordinate components $x_{k}, y_{k}$ and $z_{k}$ of arbitrary point $K$ can be determined, thus, the conclusion about angle of obstacle avoidance on critical boundary of multiple threat obstacles space VOSC can be drawn: on the feasible critical curve of UAV's obstacle avoidance, set arbitrarily obstacle avoidance turning point as a coordinate component of $K\left(x_{k}, y_{k}, z_{k}\right)$, the others can be determined by relational expression (32), (33). In this way, the expected velocity vector of the UAV's obstacle avoidance can be determined as $\hat{\boldsymbol{v}}_{u}=\boldsymbol{P}_{u} \boldsymbol{K}=$ $\left(x_{k}-x_{u}, y_{k}-y_{u}, z_{k}-z_{u}\right)$.

From this, two direction angles of vector $\boldsymbol{P}_{u} \boldsymbol{K}$ in threedimensional space can be calculated, that is, the expected velocity vector direction angles $\hat{\psi}_{u}$ and $\hat{\theta}_{u}$, rotation angles $\Delta \psi_{u}$ and $\Delta \theta_{u}$ of the corresponding UAV velocity vector $\boldsymbol{v}_{u}$ can also be calculated.

\subsection{Calculation of collision avoidance point}

\subsubsection{Calculation of collision avoidance for a single obstacle}

According to the requirements for initialization conditions of trajectory re-planning, the coordinates of required insertion point $\boldsymbol{Q}_{\text {avo }}$, that is, the obstacle's position coordinate $P_{o}^{\prime}\left(x_{o}^{\prime}, y_{o}^{\prime}, z_{o}^{\prime}\right)$ after obstacle avoidance time-interval $t$ is determined as

$$
\boldsymbol{P}_{o}^{\prime}=\left[\begin{array}{c}
x_{o}^{\prime} \\
y_{o}^{\prime} \\
z_{o}^{\prime}
\end{array}\right]=\left[\begin{array}{c}
x_{o}+\left(v_{o} \cos \theta_{o} \cos \psi_{o}\right) t \\
y_{o}+\left(v_{o} \cos \theta_{o} \sin \psi_{o}\right) t \\
z_{o}+\left(v_{o} \sin \theta_{o}\right) t
\end{array}\right]
$$

To improve the safety and reliability of the re-planning trajectory, set the obstacle avoidance insertion point to the opposite direction of the obstacle's velocity $\boldsymbol{v}_{O}$ and multiply the swelling radius of obstacle by a factor $k$, considering the obstacle's position coordinate $P_{o}^{\prime}\left(x_{o}^{\prime}, y_{o}^{\prime}, z_{o}^{\prime}\right)$ after the time-interval $t$, the coordinate of obstacle avoidance insertion point $Q_{\text {avo }}\left(x_{a v o}, y_{a v o}, z_{a v o}\right)$ is

$$
\boldsymbol{Q}_{\text {avo }}=\left[\begin{array}{c}
x_{\text {avo }} \\
y_{\text {avo }} \\
z_{\text {avo }}
\end{array}\right]=\left[\begin{array}{c}
x_{o}^{\prime}+R^{\prime} \cos \theta_{o} \cos \psi_{o} \\
y_{o}^{\prime}+R^{\prime} \cos \theta_{o} \sin \psi_{o} \\
z_{o}^{\prime}+R^{\prime} \sin \theta_{o}
\end{array}\right]
$$

where $R^{\prime}=k R, k$ is the setting constant and $k \geqslant 1$.

\subsubsection{Calculation of collision avoidance for multiple} obstacles

In velocity space, identify $m$ simultaneous obstacles $\left\{O_{1}, O_{2}, \ldots, O_{m}\right\}$ to UAV collision and the time $t_{c_{i}}(i=$ $1,2, \ldots, m)$ of collision, select the avoidance guidance time as

$$
t=\min \left(t_{c_{1}}, t_{c_{2}}, \ldots, t_{c_{m}}\right)
$$

where $t_{c_{i}}=\frac{d_{0}-R_{i}}{\left\|\boldsymbol{v}_{o_{i}}\right\|}$.

When there is collision risk between UAV and obstacles, the time of collision should be worked out before selecting the minimum avoidance time among the obstacle as the 'major' obstacle, then the coordinate of obstacle avoidance insertion point can be determined by the method adopted in single obstacle avoidance.

\subsection{General method of avoiding obstacles}

Based on the above strategies, the obstacle avoidance method based on the obstacle SC can be described as follows.

Step 1 According to the position and velocity, establish the space VOSC for the obstacle that to be avoided using the method proposed in Section 3.1. Calculate the SC's parameters by the method in Section 3.2.

Step 2 Calculate the obstacle avoidance direction. For the single obstacle, use the method in Section 4.1. For the multiple obstacles simultaneous avoidance, adopt the method in Section 4.2.

Step 3 Calculate the insertion point for obstacle avoidance. Predict the position coordinate for the insertion point.

Step 4 Based on the above calculated obstacle avoidance direction and the position coordinate of the insertion point, the UAV path can be replanned and complete the obstacle avoidance in tolerance time.

\section{Simulation and analysis of obstacle avoidance planning}

Based on the principle of three-dimensional VO avoidance, the completion of dynamic obstacle avoidance simulation can be divided into three steps, as the example of pythagorean hodograph $(\mathrm{PH})$ curve planning method [23] shown below: 
(i) Based on the PH trajectory planning algorithm, complete the trajectory planning from the initial point to the target point;

(ii) UAV flies along the PH trajectory, detects obstacles within a certain distance and justifies whether to avoid the detected obstacle;

(iii) When UAV needs to avoid the detected obstacle, the obstacle avoidance direction angle of UAV's velocity vector and the coordinate of insertion point should be worked out on the basis of the three-dimensional velocity obstacle cone method. Then complete the obstacle avoidance with the trajectory re-planning method. The new trajectory can be generated between the obstacle detected point and the interrupt point. Then take the interrupt point as the initial point and the object point as the final point, the next trajectory can also be generated. To keep the continuity of the trajectory, on the detect point and the interrupt point, the direction of the tangent line keeps constant. Based on the $\mathrm{PH}$ trajectory planning method, find a flyable $\mathrm{PH}$ path meeting the related constraint kinematics performance such as curvature. It is noteworthy that to focus more on the method performance, these simulations do not include the dynamics of the vehicles, or the effect of the environment, for example, wind and gravity.

Suppose the UAV flies from $P_{s}(0,0,0)$ to $P_{f}(500,200,100)$, the UAV velocity is $\left\|\boldsymbol{v}_{u}\right\|=50 \mathrm{~m} / \mathrm{s}$. The planned flyable PH trajectory is shown in Fig. 8.

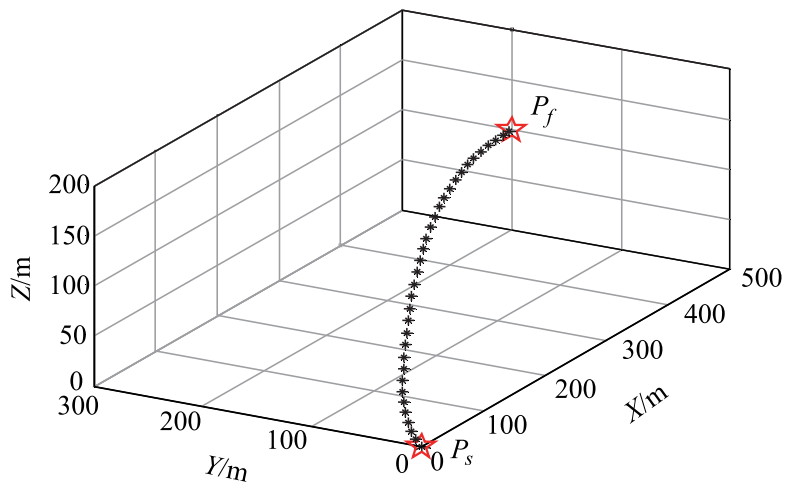

Fig. 8 Flyable PH trajectory of UAV

\subsection{Single dynamic threat obstacle avoidance}

When performing tasks in dynamic environment, as UAV detects dynamic threat obstacles in the surrounding environment, the simplest case of UAV's obstacle avoidance is that there is only one threat among the detected obstacles during the collision avoidance. The initial conditions for UAV and threat obstacles are given as Table 1. Besides, based on the relative size relationship between UAV and threat obstacle $O$, the corresponding obstacle spherical radius is $R=25$, the detection distance of UAV is $d_{0}=100 \mathrm{~m}$.

Table 1 Initial conditions for single threat obstacle avoidance

\begin{tabular}{ccc}
\hline Initial condition & UAV & $O$ \\
\hline Position coordinate & $(162,101,64.3)$ & $(254,63.6,60.7)$ \\
Velocity size & 50 & 40 \\
Velocity direction & $\left(24.7^{\circ}, 12.0^{\circ}\right)$ & $\left(135^{\circ}, 12.4^{\circ}\right)$ \\
Threat judgment & & $\alpha<\alpha_{o}$ \\
Spherical parameter & & $G(46.3,30.2,3.23,22.2)$ \\
\hline
\end{tabular}

In Fig. 8, the $\mathrm{PH}$ path between the starting point $P_{s}$ and the target point $P_{f}$ is planned, which meets the requirements of UAV's performance constraints and safety requirement for flight. When flying along the PH path, UAV detects threat obstacle $O$ in the surrounding environment, the corresponding simulation results are shown in Fig. 9.

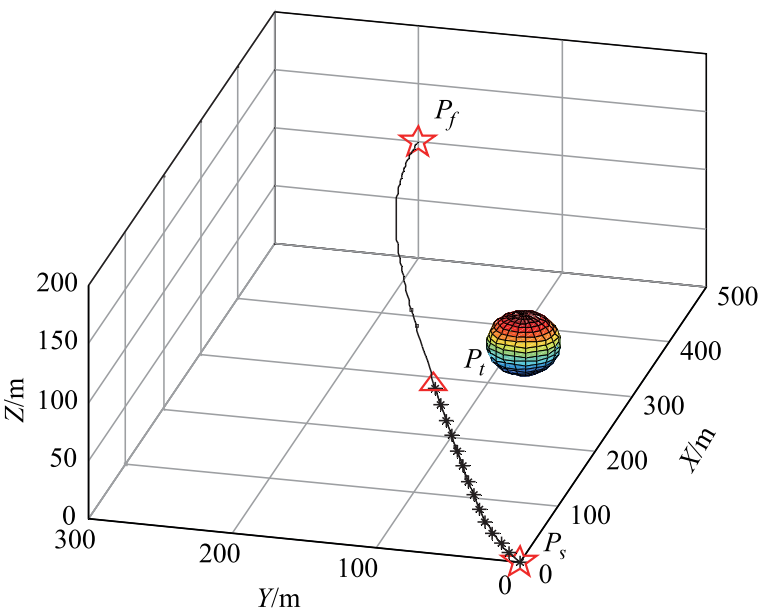

Fig. 9 Simulation diagram of UAV detecting obstacle $O$ at point $P_{t}$

From the initial conditions of UAV and obstacle in Table 1 , according to the judgment condition of collision avoidance in three-dimensional space, it can be determined that the detected obstacle is threatening and needed to be avoided, the corresponding simulation result of collision avoidance judgment is shown in Fig. 10.

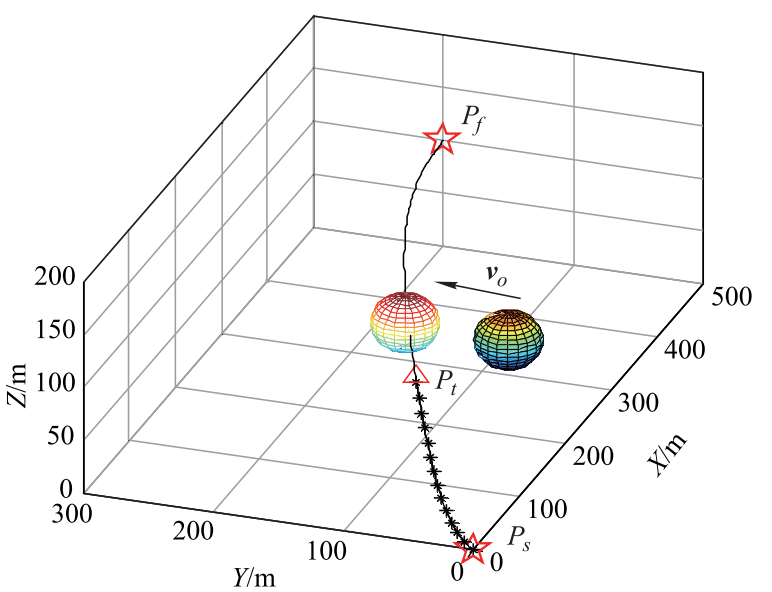

Fig. 10 Simulation diagram of obstacle avoidance judgment for detected obstacle $O$ 
According to the calculation method of space VOSC parameters in Section 3.2, the SC parameters of threat obstacle $O$ can be figured out as shown in Table 1, and the effects of the threat obstacle $O$ on UAV's flight can be quantified based on the determined spherical cap parameters. In addition, according to the calculation method of minimum obstacle avoidance velocity vector direction and collision point in single obstacle avoidance, the coordinates of the corresponding collision point $\boldsymbol{Q}_{a v o}$ and velocity vector direction angle $\left(\hat{\psi}_{u}, \hat{\theta}_{u}\right)$ of UAV can be figured out respectively, which are $(226.34,159.56,79.05),\left(36.6^{\circ}, 13.2^{\circ}\right)$. At this time, the deflected space angle of the UAV's velocity vector direction in obstacle avoidance is the minimum. Then the determined collision avoidance point and direction are taken as the initial conditions for the $\mathrm{PH}$ path planning method, the simulation results of UAV's obstacle avoidance path re-planning are shown in Fig. 11.

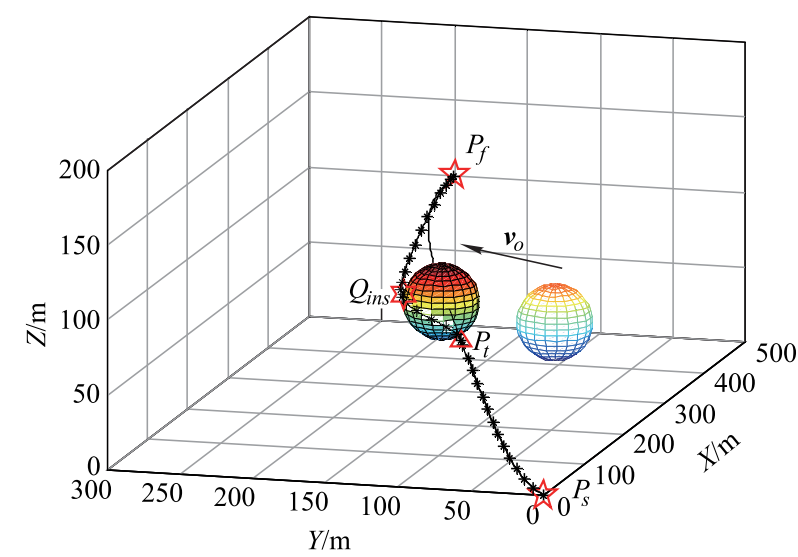

Fig. 11 Simulation diagram of UAV's single threat obstacle avoidance

The simulation result shows that the re-planned path for UAV can realize the single threat obstacle avoidance, and the path is safe and flyable.

To validate the peformance of the space spherical cap obstacle avoidance method (SCOAM), the comparison results of this method with the dimension reduction obstacle avoidance method (DROAM) in the horizontal plane (HP) and vertical plane (VP) in [19] are shown in Table 2, which indicates that the deflection angle based on space VOSC method is minimum, the path length is the shortest, and it costs the least time for obstacle avoidance.

Table 2 Comparison of different collision avoidance methods for the single obstable

\begin{tabular}{cccc}
\hline Method & $\begin{array}{c}\text { Deflection } \\
\text { angle } /\left(^{\circ}\right)\end{array}$ & $\begin{array}{c}\text { Path } \\
\text { length/m }\end{array}$ & $\begin{array}{c}\text { Obstacle } \\
\text { avoidance time/s }\end{array}$ \\
\hline SCOAM & 11.9 & 575.6 & 1.75 \\
DROAM/HP & 12.8 & 579.8 & 1.81 \\
DROAM/VP & 20.4 & 587.1 & 1.96 \\
\hline
\end{tabular}

It should be noted that in [24], the obstacle avoidance plane needed to be searched from multiple random selected planes. In [22], twelve planes were needed to find the comparatively plane. While the proposed space SCOAM can directly find the optimum obstacle avoidance plane through calculation.

\subsection{Multiple dynamic threat obstacles avoidance}

When UAV detects multiple dynamic threat obstacles, the situation is more complex. As to the multiple dynamic threat obstacles avoidance, the initial conditions for two threat obstacles are given in Table 3, and the performance of the obstacle avoidance method based on threedimensional velocity obstacle cone is simulated and verified.

Table 3 Initial conditions of multiple dynamic threat obstacles

\begin{tabular}{ccc}
\hline Initial condition & $O_{1}$ & $O_{2}$ \\
\hline Position coordinate & $(254,63.8,59.8)$ & $(208,186,42.5)$ \\
Velocity size & 35 & 32 \\
Velocity direction & $\left(106^{\circ}, 11.4^{\circ}\right)$ & $\left(-50.3^{\circ},-15.4^{\circ}\right)$ \\
Swelled radius & $R_{1}=25$ & $R_{2}=30$ \\
Threat judgment & $\alpha_{1}<\alpha_{o_{1}}$ & $\alpha_{2}<\alpha_{o_{2}}$ \\
& $G_{o_{1}}(38.1,15.4$, & $G_{o_{2}}(32.2,22.8$, \\
SC parameter & $3.10,40.4)$ & $23.5,49.9)$ \\
\hline
\end{tabular}

In the process of executing a task along the initial planned safe PH flight path, when UAV arrives at the current point $P_{t}$, the simulation results of UAV detecting two dynamic threat obstacles $O_{1}, O_{2}$, are shown in Fig. 12.

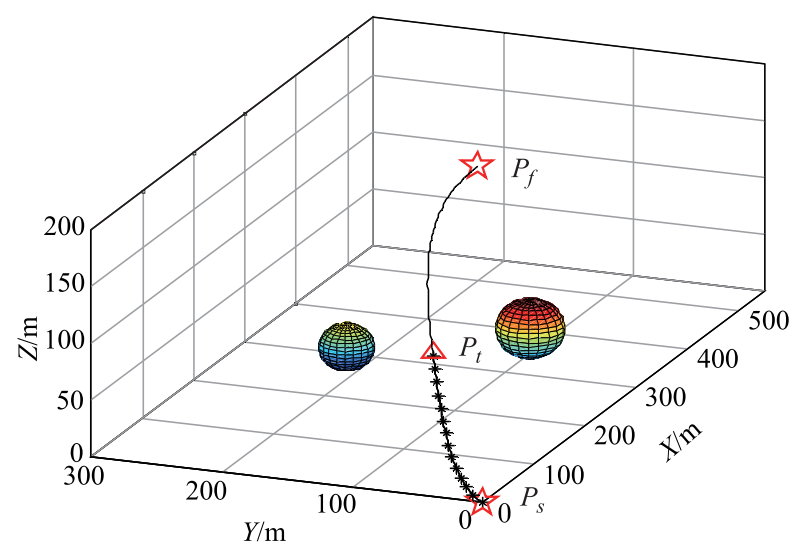

Fig. 12 Simulation diagram of UAV detecting obstacles $O_{1}, O_{2}$ at point $P_{t}$

Based on the threat judgment method and the initial conditions of obstacles and UAV, it can be obtained that obstacles $O_{1}, O_{2}$ meet the UAV threat judgment conditions $\alpha_{1}<\alpha_{o_{1}}, \alpha_{2}<\alpha_{o_{2}}$ simultaneously, and two obstacles in Fig. 12 are threatening. Then UAV needs to avoid them simultaneously, this situation belongs to multiple dynamic threat obstacles avoidance, the corresponding simulation results are shown in Fig. 13. 


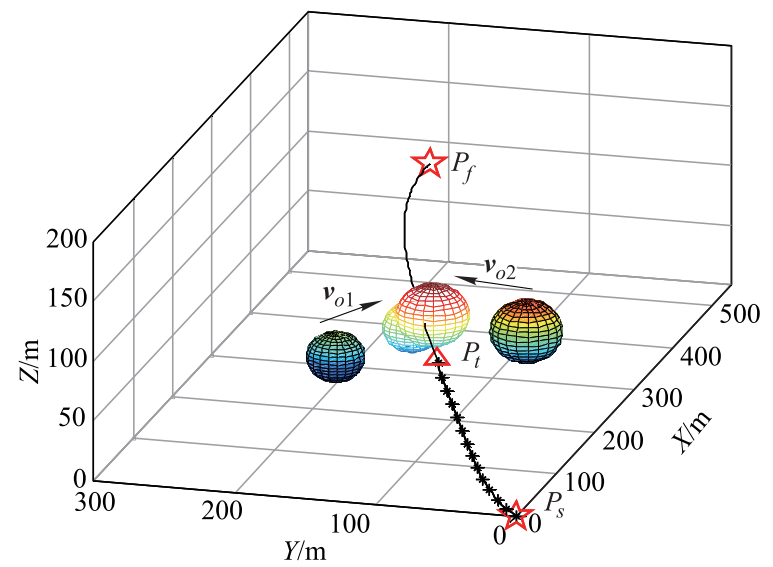

Fig. 13 Judgment simulation diagram of avoiding detected obstacles $\mathrm{O}_{1}, \mathrm{O}_{2}$

According to the calculation method of space VOSC parameters, the parameters of two dynamic threat obstacles $O_{1}$ and $O_{2}$ can be obtained as shown in Table 3.

According to the three-dimensional VO avoidance method, the position coordinates of multiple dynamic threat obstacles avoidance point can be determined as $Q_{\text {avo }}(229,148,77.6)$. Select the point $K$ on the obstacle avoidance boundary as the critical state point for obstacle avoidance, whose coordinate is $(206.5,101.0,88.5)$, velocity vector direction angle $\left(\hat{\psi}_{u}, \hat{\theta}_{u}\right)$ is $\left(22.2^{\circ},-38.9^{\circ}\right)$ and the deflection angle $\left(\Delta \psi_{u}, \Delta \theta_{u}\right)$ is $\left(-3.88^{\circ}, 12.86^{\circ}\right)$. Then taking the determined collision avoidance point and the velocity vector deflection direction as the initial conditions of UAV's obstacle avoidance and path re-planning, the initial trajectory is re-planed, which is show in Fig. 14.

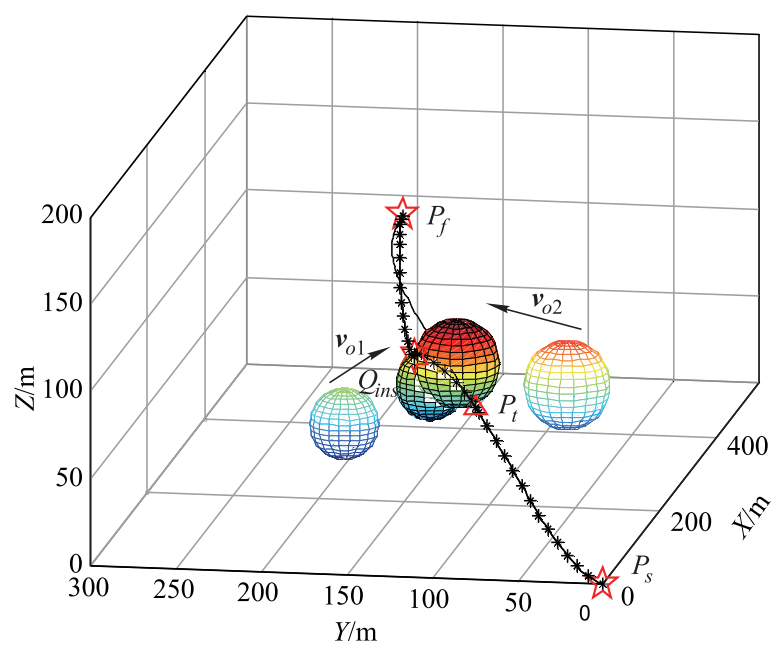

Fig. 14 Simulation diagram of UAV's multiple obstacles avoidance

The simulation results show that UAV's re-planed path can realize the multiple dynamic threat obstacles avoidance. Meanwhile, the space VOSC quantifies the effects of dynamic threat obstacles on the UAV's obstacle avoidance, simplifies the analysis and solution of velocity vector direction in multiple dynamic threat obstacle avoidance. The determination of velocity vector direction for obstacle avoidance based on mission requirements and UAV's performance index increases the velocity vector direction selectivity at collision avoidance point.

In addition, the space SCOAM in this paper is compared with the DROAM in [19] in the HP and VP, the results are shown in Table 4, which indicates the superiority of the space SCOAM. The proposed method carries out the corresponding maneuver simultaneously on the HP and the VP, which improves the flexibility of obstacles avoidance, and can easily meet the dynaminc performance constraint, such as the overload.

Table 4 Comparison of different collision avoidance methods for the multiple obstacles

\begin{tabular}{cccc}
\hline Method & $\begin{array}{c}\text { Deflection } \\
\text { angle } /\left(^{\circ}\right.\end{array}$ & $\begin{array}{c}\text { Path } \\
\text { length/m }\end{array}$ & $\begin{array}{c}\text { Obstacle } \\
\text { avoidance time/s }\end{array}$ \\
\hline SCOAM & 13.4 & 582.4 & 1.19 \\
DROAM/HP & 25.5 & 595.7 & 1.45 \\
DROAM/VP & 18.4 & 588.2 & 1.28 \\
\hline
\end{tabular}

The potential field method [7] implemented based on the imminent obstacle avoidance can cause disturbance and is not easy to avoid multiple obstacles. This indicates that the SC method is more efficient in the resulting avoidance path.

\section{Conclusions}

This paper described a novel three-dimensional VO method for conflict resolution. The method takes simultaneous multiple dynamic obstacles into account and can generate a reactive three-dimensional avoidance maneuver direction for a UAV to resolve conflicts. Through prediction, the insertion point position of UAV re-planed flight path for the obstacles avoidance is obtained. With this insertion point position and avoidance velocity vector direction for obstacles avoidance, the flight path of UAV is replanned. Simulation results have demonstrated the performance of the three-dimensional VO method.

The performance of the method has three key features: the concept of VOSC, the direct three-dimensional avoidance velocity vector arbitrary change, the insertion point prediction for the obstacle avoidance.

The concept of VOSC is introduced through the expression of four SC parameters, by which the effects of dynamic threat obstacles on UAV's collision avoidance can be quantified and the simultaneous multiple dynamic obstacles avoidance critical curve can be given. The direct three-dimensional avoidance velocity vector arbitrary change method of any obstacle avoidance point on the avoidance critical curve is provided, which can satisfy the different UAV's task requirements and its performance, and enhance the flexibility of obstacle avoidance. Through 
prediction, the insertion point for the obstacle avoidance can be obtained and the flight path can be re-planned.

\section{References}

[1] REBERTO C, DAVID A, JOSE A C, et al. Conflict detection and resolution method for cooperating unmanned aerial vehicles. Journal of Intelligent \& Robotic Systems, 2012, 65(1-4): $495-505$.

[2] PEHLIVANOGLU Y V. A new vibrational genetic algorithm enhanced with a Voronoi diagram for path planning of autonomous UAV. Aerospace Science \& Technology, 2012, 16(1): $47-55$.

[3] ZHANG Y, GONG D W, ZHANG J H. Robot path planning in uncertain environment using multi-objective particle swarm optimization. Neurocomputing, 2013, 103(2): 172-185.

[4] GENG N, GONG D W, ZHANG Y. PSO-based robot path planning for multi-survivor rescue in limited survival time. Mathematical Problems in Engineering, 2014(2), DOI: 10.1155/2014/187370.

[5] GENG N, GONG D, ZHANG Y. Robot path planning in an environment with many terrains based on interval multi-objective PSO. International Journal of Robotics and Automation, 2016, 31(2): $100-110$.

[6] YAO P, WANG H L, SU Z K. Real-time path planning of unmanned aerial vehicle for target tracking and obstacle avoidance in complex dynamic environment. Aerospace Science and Technology, 2015, 47: 269-279.

[7] CHEN Y, LUO G, MEI Y, et al. UAV path planning using artificial potential field method updated by optimal control theory. International Journal of Systems Science, 2016, 47(6): $1407-$ 1420.

[8] WILKIE D, BERG J V D, MANOCHA D. Generalized velocity obstacles. Proc. of the IEEE/RSJ International Conference on Intelligent Robots and Systems, 2009: 5573-5578.

[9] ALEJO D, COBANO J A, TRUJILLO M A, et al. The speed assignment problem for conflict resolution in aerial robotics. Proc. of the IEEE International Conference on Robotics and Automation, 2012: 3919-3424.

[10] LAUDERDALE T. Probabilistic conflict detection for robust detection and resolution. Proc. of the AIAA Aviation Technology, Integration, and Operations, 2012: AIAA 2012-5643.

[11] JENIE Y I, VAN KAMPEN E J, DE VISSER C C, et al. Selective velocity obstacle method for deconflicting maneuvers applied to unmanned aerial vehicles. Journal of Guidance, Control, and Dynamics, 2015, 38(6): 1140-1145.

[12] SHIN H S, WHITE B A, TSOURDOS A. UAV conflict detection and resolution for static and dynamic obstacles. Proc. of AIAA Guidance, Navigation, and Control Conference, 2008: AIAA 2008-6521.

[13] SHIN H S, TSOURDOS A, WHITE B A. UAS conflict detection and resolution using differential geometry concepts. sense and avoid in UAS: research and applications. Hoboken: Wiley, 2012.

[14] WHITE B A, SHIN H S, TSOURDOS A. UAV obstacle avoidance using differential geometry concepts. Proc. of IFAC World Congress, 2011: 6325-6330.

[15] ANUSHA M, RADHAKENT P. Reactive collision avoidance using nonlinear geometric and differential geometric guidance. Journal of Guidance, Control, and Dynamics, 2011, 34(1): $303-310$.

[16] CHEN H D, CHANG K C, CRAIG S A. UAV path planning with tangent-plus-Lyapunov vector field guidance and obstacle avoidance. IEEE Trans. on Aerospace and Electronic Systems, 2013, 49(2): 840 - 856 .

[17] MACHADO P, BOUSSON K. Automatic collision avoidance system based on geometric approach applied to multiple air- craft. Proc. of the International Conference for Research in Air Transportation, 2014: 272418676.

[18] HAN S C, BANZ H, YOO C S. Proportional navigation-based collision avoidance. International Journal of Control, Automation, and Systems, 2009, 7(4): 553-565.

[19] BELKHOUCHE F, BENDJILALI B. Reactive path planning for 3-d autonomous vehicles. IEEE Trans. on Control Systems Technology, 2012, 20(1): 249-256.

[20] WATANABLE Y, CALISE A, JOHNSON E N. Minimum effort guidance for vision-based collision avoidance. Proc. of the AIAA Atmospheric Flight Mechanics Conference and Exhibit, 2006: 589-601.

[21] YAZDI I J, KAMPEN E J V, COEN C D V, et al. Threedimensional velocity obstacle method for UAV uncoordinated avoidance maneuvers. Proc. of the AIAA Guidance, Navigation, and Control Conference, 2016: AIAA 2016-1629.

[22] YAZDI I J, KAMPEN E J V, COEN C D V, et al. Three-dimensional velocity obstacle method for uncoordinated avoidance maneuvers of unmanned aerial vehicles. Journal of Guidance, Control, and Dynamics, 2016, 39(10): 2312 2323.

[23] ZHANG Y, YANG X X, ZHOU W W, et al. Study of three-dimensional on-line path planning for UAV based on Pythagorean hodograph curve. International Journal on Smart Sensing and Intelligent System, 2015, 8(4): 1641-1666.

[24] LORENZ S, WALTER F. Collision-avoidance framework for small fixed-wing unmanned aerial vehicles. Journal of Guidance, Control, and Dynamics, 2014, 37(4): 1323 - 1328.

\section{Biographies}

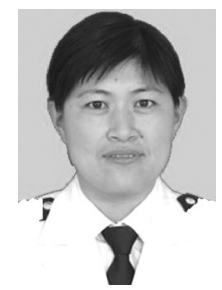

YANG Xiuxia was born in 1975. She received her Ph.D. degree in electrical engineering from Naval University of Engineering, Wuhan, China in 2005. Since 2000, she has been with Department of Control Engineering of Naval Aeronautical and Astronautical University, where she is currently a vice professor of Naval Aviation University. Her main research interests include nonlinear control theory with applications to robots, aircraft and other mechanical systems. E-mail: yangxiuxia@126.com

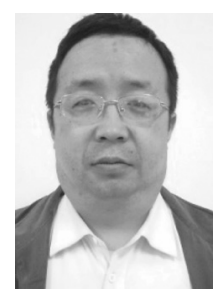

ZHANG Yi was born in 1971. He received his Master degree in control theory and application from Naval Aeronautical and Astronautical University, Yantai, China in 2001. Since 2000, he has been with Department of Control Engineering of Naval Aeronautical and Astronautical University, where he is currently a vice professor of Naval Aviation University. His main research interests include nonlinear control theory with applications to robots, aircraft and other mechanical systems.

E-mail: changyee@tom.com

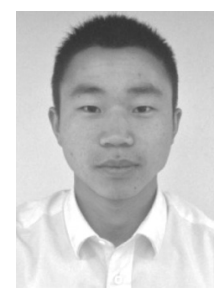

chanical systems.
ZHOU Weiwei was born in 1991. He received his Bachelor degree in test and control engineering from Naval Aeronautical and Astronautical University, Yantai, China in 2014. He is currently working toward his Master degree in control theory and application at Naval Aviation University. His main research interests include nonlinear control theory with applications to robots, aircraft and other me-
E-mail: zhouweiwei8181@sina.com 